\title{
METODOS PARA EL ESTABLECIMIENTO DE PLANTACIONES DE Eucalyptus camaldulensis Y Quillaja saponaria EN LA ZONA ARIDA DE CHILE
}

Johannes Wrann H. $\left({ }^{*}\right)$

Pedro Infante L. $\left({ }^{* *}\right)$

\section{RESUMEN}

En la zona árida de Chile, en la provincia de Choapa, IV Región, con unos $215 \mathrm{~mm}$ de precipitación anual, se probaron tres tratamientos de preparación de suelo, con y sin aplicación de fertilización y control de malezas, con el fin de determinar su efecto en el establecimiento de plantaciones de Eucalyptus camaldulensis y Quillaja saponaria.

Los resultados indican que el factor individual más importante es el control de la vegetación competitiva, que permite asegurar la supervivencia y crecimiento inicial de las plantaciones.

La fertilización afecta positivamente el desarrollo de las plantas, si se aplica en conjunto con el herbicida. Al no combinar ambas aplicaciones baja la sobrevivencia de las plantas por un mayor desarrollo de la competencia.

Los mejores resultados se obtuvieron al combinar la preparación del suelo con subsolado y la aplicación de fertilizante (NPK) con control de la vegetación competidora, lográndose resultados altamente satisfactorios, en consideración a las características de la zona de estudio.

\section{ABSTRACT}

Soil preparation treatments, weed control and fertilization practices in a Eucalyptus camaldulensis and Quillaja saponaria plantation were tested.

The field trial is located in Chile's arid region with $215 \mathrm{~mm}$ annual rainfall.

Weed control is the most important factor to assure high survival and first growth of the plantation.

Growth is positively influenced with fertilizing together with a weed control, otherwise a negative survival effect is caused.

Best results are achieved with intensive establishment practices, combining ripping, fertilizing and weed control. In consideration of the environmental conditions, highly satisfactory results have been achieved.

(*) : Ingeniero Forestal. División Silvicultura. Instituto Forestal. Huérfanos 554 - Piso 4. Santiago Chile.

$\left({ }^{* *}\right)$ : Ingeniero Forestal. Consultora de Ingenieria Forestal Ltda. Santiago - Chile 


\section{INTRODUCCION}

La zona centro norte del país presenta serias limitantes para el establecimiento de plantaciones forestales. Esto se debe a las escasas precipitaciones, el largo período de sequía en el año y a los suelos con fertilidad limitada y con algún grado de erosión. Por esta razón, se considera de interés ensayar técnicas de establecimiento tales, que permitan obtener una respuesta favorable en la supervivencia y crecimiento inicial, para las especies forestales consideradas como aptas para la región.

Basado en experiencias sobre técnicas de establecimiento con especies de rápido crecimiento (BODEN, 1984; CROMER, 'R.N., 1984; BACKSTROM, 1981, SCHONAU et al 1981; SCHONAU, 1984) y considerando las especies seleccionadas a través del proyecto "Introducción de Especies Forestales" de INFOR (CORFO - INFOR, 1986), se instalaron 11 ensayos de métodos de plantación dentro del período 1984-87 en la zona árida y semiárida del país. La primera de estas experiencias fue establecida y analizada por PRADO y ROJAS (1987), obteniéndose resutados muy alentadores. Dicho ensayo considera distintos tratamientos al suelo, reducción de competencia y fertilización en una plantación de Eucalyptus globulus establecida en 1984 en la zona central del país. A la edad de 2 años se estimó una biomasa diez veces superior en el tratamiento más intenso, con respecto al sistema de plantación tradicional (hoyo).

El objetivo del ensayo que aquí se describe es analizar el crecimiento inicial de Eucalyptus camaldulensis y Quillaja saponaria en la zona árida del país (interior de la IV Región), según diferentes métodos de plantación: preparación del suelo, control de competencia por eliminación de malezas y aplicación de fertilizantes.

\section{MATERIAL Y METODO}

\section{Descripción del lugar de ensayo}

El ensayo se ubica en la IV Región, comuna de Illapel a aproximadamente $10 \mathrm{Km}$ al S.W. de la ciudad del mismo nombre, en el predio "Bellavista", con acceso por el camino antiguo Illapel - Los Vilos. El ensayo está ubicado en un terreno de aptitud ganadero-forestal con una pendiente de hasta un $20 \%$ y exposición predominante N.E. El suelo es de origen granítico con una profundidad de $60-80 \mathrm{~cm}$ sobre el material generador. La textura del horizonte superior es franco arcillo arenosa. El contenido de materia orgánica es bajo, pero la disponibilidad de nitrógeno, fósforo, potasio y boro no restringen el crecimiento de las plantas, N-P-K-B horizonte superior $=13-17-382-0,93 \mathrm{ppm}$ respectivamente $($ TORO, J., 1986). La textura y la densidad aparente $\left(1,35-1,40 \mathrm{~g} / \mathrm{cm}^{3}\right.$ en el horizonte superior) y un pH de 7,2 - 7,5 son adecuados para el crecimiento de algunas especies de Eucalyptus (TORO, J., 1986).

El clima corresponde al tipo de Estepa con Gran Sequedad Atmosférica "BST" según Köeppen (FUENZALIDA, H., 1967) o al tipo Mediteráneo Arido según Emberger (DI CASTRI y HAJEK, 1976). Se caracteriza por una precipitación invernal que en promedio alcanza $215 \mathrm{~mm}$ anuales y un largo período seco, de 8 a 11 meses por año.

La vegetación natural existente en el área es la estepa de Acacia caven. En el lugar de ensayo el estrato arbustivo bajo está dominado por Gutierrezia paniculata ("Pichanilla".) 


\section{Tratamientos y Diseño Experimental}

El ensayo se estableció en el invierno de 1985. Las plantas fueron producidas en macetas (siembra en primavera 1984) en el vivero de INFOR en Santiago para la especie Quillaja saponaria y en el vivero de CONAF - Melipilla para la especie Eucalyptus camaldulensis. Esto significa que las plantas tuvieron que transportarse $280 \mathrm{Km}$ ó $347 \mathrm{Km}$ según procedían del vivero de Santiago o de Melipilla respectivamente.

Como protección contra animales herbívoros, se colocó a cada planta una barrera de lata con agujeros de aproximadamente $2.5 \mathrm{~cm}$. de diámetro (desecho de la fabricación de tapas de botellas). Su altura es de aprox. $50 \mathrm{~cm}$.

Debido a la sequía del año 1985 hubo una alta mortalidad de plantas en ambas especies, que alcanzó a un $28,6 \%$ en Quillaja saponaria y un $32,2 \%$ en Eucalyptus camaldulensis. Por este motivo se efectuó un replante en Junio de 1986.

La plantación se regó en las siguientes fechas : Julio 1985 (inmediatamente después de la plantación); Septiembre 1985; Enero 1986; Febrero 1986; Noviembre 1986 y Enero 1987. La cantidad de agua suministrada por planta fue de 21 en el primer riego y de 41 en cada uno de los restantes.

En el verano del primer año se observaron cortes de ápices producidos por liebres en las plantas de Eucalypto. Por tal motivo se aplicó un repelente (Pomarsol F - Bayer i.a. Thiuram TMTD $80 \%$ ) en solución con agua al $12,5 \%$ conc. y se aplicó en una línea alrededor de cada bloque. Se efectuaron tres aplicaciones distribuidas entre el verano 1986 y el de 1987.

Los tratamientos del ensayo se hicieron según un diseño factorial $3 \times 2 \times 2$, con tres repeticiones, que combinan los siguientes factores :

A. Preparación de Suelo

$$
\begin{aligned}
& 0 \text { : Hoyo } \\
& 1 \text { : Surco } \\
& 2 \text { : Subsolado }
\end{aligned}
$$

B. Fertilización

$$
\begin{aligned}
& 0: \text { Testigo (sin aplicación) } \\
& 1: 170 \mathrm{~g} \mathrm{NPK} \text { por planta }
\end{aligned}
$$

\section{Control de Competencia}

$$
\begin{aligned}
& 0: \text { Testigo (sin control) } \\
& 1 \quad \text { Limpia manual }
\end{aligned}
$$

De la combinación de estos factores resultan los siguientes tratamientos :

$\mathrm{T}_{1}$ : Hoyo de $30 \mathrm{~cm} \times 30 \mathrm{~cm} \times 30 \mathrm{~cm}$ hecho con chuzo o picota y pala, que representa el tratamiento menos intensivo del suelo y que ha sido el método tradicionalmente aplicado en Chile para la plantación en esta región.

$\mathrm{T}_{2}$ : Hoyo - Fertilizante. El tratamiento anterior, pero con aplicación de fertilizante un par de semanas después de la plantación.

$\mathrm{T}_{3}$ : Hoyo - Control competencia. El tratamiento $\mathrm{T} 1$ con control de la competencia mediante limpia manual.

$\mathrm{T}_{4}$ : Hoyo - Fertilizante - Control competencia. La combinación de los tres anteriores. 
$\mathrm{T}_{\mathrm{s}}$ : Surco hecho con arado liviano tirado por tractor, que es el otro método normalmente empleado en la región árida - semiárida para establecer plantaciones forestales.

$\mathrm{T}_{6}$ : Surco - Fertilizante

$T_{i}$ : Surco - Control competencia

$T_{8}$ : Surco - Fertilizante - Control competencia

$\mathrm{T}_{9}$ : Subsolado a $40 \mathrm{~cm}$ de profundidad, hecho con arado subsolador tirado por un tractor agrícola.

$\mathrm{T}_{10}$ : Subsolado - Fertilizante

$T_{11}$ : Subsolado - Control competencia

$T_{12}$ : Subsolado - Fertilizante - Control competencia

Estos 12 tratamientos (repeticiones) fueron distribuidos en bloques ubicados en forma perpendicular a la pendiente para eliminar las variaciones que éstas produce.

Debido a la configuración topográfica del terreno, uno de los bloques de cada especie quedó expuesto al viento S.W. predominante, siendo para Quillaja saponaria la ubicación más desfavorable. Por esta causa se produjo una mortalidad importante, lo que obligó a proteger las plantas después del replante al año siguiente. Esta protección consistió en una pequeña barrera de la altura de la planta, con ramas de arbusto del lugar, afirmadas con alambre dispuesto entre dos estaquillas.

La fertilización se realizó dos a cuatro semanas después de la plantación a fines de invierno. A cada planta se aplicó $210 \mathrm{~g}$ de fertilizante : $50 \mathrm{~g}$ de superfosfato triple $(20,1 \% \mathrm{P})$; $50 \mathrm{~g}$ de sulfato de potasio $(50 \% \mathrm{~K})$ y $110 \mathrm{~g}$ de urea $(46 \% \mathrm{~N})$. La mezcla se distribuyó en dos pequeñas zanjas hechas a ambos lados de la planta a unos $20 \mathrm{~cm}$ de éstas en el mismo sentido de la pendiente.

Al año siguiente en la misma época se realizó una aplicación idéntica de fertilizante.

Es necesario aclarar que este ensayo no tenía como objetivo recomendar una tasa óptima de fertilización, sino evaluar el crecimiento de la especie con un suplemento de nutrientes.

El control de la competencia se realizó en la misma época de la aplicación del fertilizante. Consistió en la limpia manual de la maleza en un radio de aprox. $1 \mathrm{~m}$ alrededor de la planta. Esta se repitió en la misma época al año siguiente (1986).

La unidad muestreal es una parcela de 25 plantas con un distanciamiento de $3 \mathrm{~m} \mathrm{x} 3 \mathrm{~m}$. El número de repeticiones es de 3 .

La medición de la supervivencia, altura total de la planta (con regla) y el diámetro en el cuello de la planta o diámetro basal (con piedemetro) se realizó en Abril de 1987, a la edad de 2 años.

\section{Metodología de Análisis}

El análisis de los resultados de adaptación para ambas especies se realizó en base a la supervivencia (con y sin replante) a la altura, diámetro en la base del tallo (diámetro basal) y al "índice de crecimiento total", que es un estimador de la biomasa. Corresponde al diámetro basal promedio al cuadrado por la altura promedio de cada parcela (DB2 $\times$ H) a la edad de dos años.

Los valores promedios de los factores y de los tratamientos se sometieron a un análisis de varianza factorial, con el fin de determinar la existencia de diferencias estadísticamente significativas entre bloques y tratamientos, y la interacción de éstos.

En consideración a que un replante puede ser necesario en esta región y para contrarrestar 
las pérdidas en las parcelas expuestas al viento, se analizó la supervivencia y el crecimiento incluyendo el replante.

Con el objeto de evaluar además el efecto de un año seco (1985) en la plantación, también se analizó la supervivencia sin incluir el replante.

\section{RESULTADOS}

Los resultados de los ensayos a la edad de dos años de Eucalyptus camaldulensis y Quillaja saponaria se presenta en los Cuadros 1 y 2 respectivamente. En éstos se entregan los valores medios de las variables empleadas para el análisis de cada uno de los tratamientos. En la supervivencia se anotan los valores con y sin replante al primer año.

\section{CUADRO 1}

\section{RESUMEN RESUltados AL $2^{\circ}$ AÑO DESPUES DE LA PLANTACION ESPECIE: Eucalyptus camaldulensis.}

\begin{tabular}{|c|c|c|c|c|c|c|c|}
\hline \multirow[b]{2}{*}{$\begin{array}{l}\text { Factor A } \\
\text { Preparación } \\
\text { del Suelo }\end{array}$} & \multirow[b]{2}{*}{$\begin{array}{l}\text { Factores B y C } \\
\text { Fertilizante- } \\
\text { Herbicida }\end{array}$} & \multirow[b]{2}{*}{ Tratamiento } & \multicolumn{2}{|c|}{ SUPERVIVENCIA \% } & \multirow[b]{2}{*}{$\begin{array}{l}\text { Alt. Prome- } \\
\text { dio (H) } \\
\text { (m) (1) }\end{array}$} & \multirow[b]{2}{*}{$\begin{array}{l}\text { Diam. en la } \\
\text { Base Prome- } \\
\text { dio (DB) } \\
\text { (cm })(1)\end{array}$} & \multirow[b]{2}{*}{$\begin{array}{c}\text { Indice de } \\
\text { Crecimien- } \\
\text { to Promed. } \\
\text { DB' } \\
\left(\mathrm{cm}^{3}\right)(1)\end{array}$} \\
\hline & & & $\begin{array}{c}\text { Sin } \\
\text { Replante }\end{array}$ & $\begin{array}{c}\text { Con } \\
\text { Replante }\end{array}$ & & & \\
\hline$A_{0}$ Hoyo & $\begin{array}{l}\mathrm{B}_{0} \mathbf{C}_{0} \text {. Testigo } \\
\mathrm{B}_{1} \mathrm{C}_{0}: \text { Fertilizante } \\
\mathrm{B}_{0} \mathrm{C}_{1}: \text { Herbicida } \\
\mathrm{B}_{1} \mathrm{C}_{1}: \text { Fert - Herb }\end{array}$ & $\begin{array}{l}\text { 1) } \mathrm{Ho} \\
\text { 2) } \mathrm{Ho} \mathrm{Fe} \\
\text { 3) } \mathrm{Ho} \mathrm{He} \\
\text { 4) } \mathrm{Ho} \mathrm{Fe} \mathrm{He}\end{array}$ & $\begin{array}{r}43 \\
7 \\
76 \\
57\end{array}$ & $\begin{array}{l}56 \\
17 \\
97 \\
87\end{array}$ & $\begin{array}{l}0.54 \\
0,52 \\
0,74 \\
0,83\end{array}$ & $\begin{array}{l}0.8 \\
0.9 \\
1,2 \\
1,3\end{array}$ & $\begin{array}{r}35,41 \\
57,62 \\
109,12 \\
175,88\end{array}$ \\
\hline TOTAL $A_{0}$ & & & 46 & 64 & 0,66 & 1,0 & 94,53 \\
\hline$A_{1}$ Surco & $\begin{array}{l}\mathrm{B}_{0} \mathrm{C}_{0}: \text { Testigo } \\
\mathrm{B}_{1} \mathrm{C}_{0}: \text { Fertilizante } \\
\mathrm{B}_{0} \mathrm{C}_{1}: \text { Herbicida } \\
\mathrm{B}_{1} \mathrm{C}_{1}: \text { Fert }- \text { Herb }\end{array}$ & $\begin{array}{l}\text { 5) } \mathrm{Su} \\
\text { 6) } \mathrm{Su} \mathrm{Fe} \\
\text { 7) } \mathrm{Su} \mathrm{He} \\
\text { 8) } \mathrm{Su} \mathrm{Fe} \mathrm{He}\end{array}$ & $\begin{array}{l}41 \\
31 \\
51 \\
65\end{array}$ & $\begin{array}{l}56 \\
59 \\
96 \\
97\end{array}$ & $\begin{array}{l}0.54 \\
0.59 \\
0.66 \\
0.89\end{array}$ & $\begin{array}{l}0.8 \\
0,8 \\
1,0 \\
1,5\end{array}$ & $\begin{array}{r}41,76 \\
41,67 \\
70,49 \\
230.99\end{array}$ \\
\hline TOTAL A , & & & 47 & 77 & 0,67 & 1,0 & 96,23 \\
\hline$A_{2}$ Subrolado & $\begin{array}{l}\mathbf{B}_{0} \mathbf{C}_{0}: \text { Testigo } \\
\mathbf{B}_{1} \mathbf{C}_{0} \text { Fertilizante } \\
\mathbf{B}_{0} \mathbf{C}_{1}: \text { Herbicida } \\
\mathrm{B}_{1} \mathbf{C}_{1}: \text { Fert }- \text { Herb }\end{array}$ & $\begin{array}{l}\text { 9) } \mathrm{Sb} \\
\text { 10) } \mathrm{Sb} \mathrm{Fe} \\
\text { 11) } \mathrm{Sb} \mathrm{He} \\
\text { 12) } \mathrm{Sb} \mathrm{Fe} \mathrm{He}\end{array}$ & $\begin{array}{l}40 \\
40 \\
61 \\
76\end{array}$ & $\begin{array}{r}73 \\
55 \\
97 \\
100\end{array}$ & $\begin{array}{l}0.60 \\
0.58 \\
0.73 \\
0.94\end{array}$ & $\begin{array}{l}0.9 \\
0.9 \\
1.2 \\
1.7\end{array}$ & $\begin{array}{r}49.37 \\
51.75 \\
104.10 \\
340.53\end{array}$ \\
\hline TOTAL. $A_{2}$ & & & 54 & 81 & 0,71 & 1,2 & 136,44 \\
\hline $\begin{array}{l}\text { TOTALES } \\
\text { FACTORES } \\
\text { B y C } \\
\text { (Todos los mét } \\
\text { de prep. de suelo) }\end{array}$ & $\begin{array}{l}B_{0} C_{0}: \text { Testigo } \\
B_{1} C_{0}: \text { Fertilizante } \\
B_{0} C_{1}: \text { Herbicida } \\
B_{1} C_{1}: \text { Fert - Herb }\end{array}$ & $\begin{array}{rrr}1 & -5 & -9 \\
2 & -5 & -10 \\
3 & -7 & -11 \\
4 & -8 & -12\end{array}$ & $\begin{array}{l}41 \\
26 \\
63 \\
66\end{array}$ & $\begin{array}{l}62 \\
44 \\
97 \\
95\end{array}$ & $\begin{array}{l}0,56 \\
0,56 \\
0,71 \\
0,89\end{array}$ & $\begin{array}{l}0,8 \\
0,9 \\
1,1 \\
1,5\end{array}$ & $\begin{array}{l}42.18 \\
50.38 \\
94,57 \\
249,13\end{array}$ \\
\hline
\end{tabular}

NOTA (1): Se incluye replante al primer año./ Ho: Hoyo; Su: Surco; Sb: Subsolado; Fe: fertilizante; He: herbicida. 
Además se presentan los valores totales de los niveles de cada factor. Por ejemplo, "Total A0", representa los promedios del tratamiento de suelo $A_{\|}$(Hoyo), cualquiera sea el nivel de los factores B y C. Estos resultados se presentan en forma gráfica en las Figuras 1-2-5-7 para la especie Eucalyptus camaldulensis y figuras 3-4-6-8 para Quillaja saponaria.

Los niveles de significancia para los distintos factores y su interacción se indican en los Cuadros 3 y 4 para Eucalyptus camaldulensis y Quillaja saponaria, respectivamente. Las diferencias significativas entre los bloques, tratamientos e interacciones entre éstos, se establecieron mediante análisis de varianza factorial de las variables supervivencia (con y sin replante), altura, diámetro basal e índice de crecimiento medio.

\section{CUADRO 2}

\section{RESUMEN RESUltados AL $2^{\circ}$ AÑo DESPUES DE LA PLANTACiON ESPECIE: Quillaja saponaria}

\begin{tabular}{|c|c|c|c|c|c|c|c|}
\hline \multirow[b]{2}{*}{$\begin{array}{c}\text { Factor A } \\
\text { Preparación } \\
\text { del Suelo }\end{array}$} & \multirow[b]{2}{*}{$\begin{array}{c}\text { Factores B y C } \\
\text { Fertilizante- } \\
\text { Herbicida }\end{array}$} & \multirow[b]{2}{*}{ Tratamiento } & \multicolumn{2}{|c|}{ SUPERVIVENCIA $\%$} & \multirow[b]{2}{*}{$\begin{array}{l}\text { Alt. Prome. } \\
\text { dio (H) } \\
\text { (m) (1) }\end{array}$} & \multirow[b]{2}{*}{$\begin{array}{l}\text { Diam. en la } \\
\text { Base Prome- } \\
\text { dio (DB) } \\
\text { (cm } k 11\end{array}$} & \multirow[b]{2}{*}{$\begin{array}{c}\text { Indice de } \\
\text { Crecimien. } \\
\text { to Promed. } \\
\mathrm{DB}^{2} \mathrm{H} \\
\left(\mathrm{cm}^{3}\right)(1)\end{array}$} \\
\hline & & & $\underset{\text { Replante }}{\text { Sin }}$ & $\begin{array}{c}\text { Con } \\
\text { Replante }\end{array}$ & & & \\
\hline$A_{0}$ Hoyo & $\begin{array}{l}\mathrm{B}_{0} \mathrm{C}_{0} \text { Testigo } \\
\mathrm{B}_{1} \mathrm{C}_{0}=\text { Fertilizante } \\
\mathrm{B}_{0} \mathrm{C}_{1}: \text { Herbicida } \\
\mathrm{B}_{1} \mathrm{C}_{1}: \text { Fert } \text { Herb }\end{array}$ & $\begin{array}{l}\text { 1) } \mathrm{Ho} \\
\text { 2) } \mathrm{Ho} \mathrm{Fe} \\
\text { 3) } \mathrm{Ho} \mathrm{He} \\
\text { 4) } \mathrm{Ho} \mathrm{Fe} \mathrm{He}\end{array}$ & $\begin{array}{l}51 \\
64 \\
99 \\
93\end{array}$ & $\begin{array}{c}59 \\
65 \\
100 \\
100\end{array}$ & $\begin{array}{l}0.27 \\
0.34 \\
0.49 \\
0.60\end{array}$ & $\begin{array}{l}0.4 \\
0.5 \\
0.8 \\
0.8\end{array}$ & $\begin{array}{c}6.66 \\
9.37 \\
35.51 \\
54.86\end{array}$ \\
\hline TOTAL $A_{0}$ & & & 77 & 81 & 0,42 & 0.6 & 26.60 \\
\hline$A_{1}$ Surco & $\begin{array}{l}\mathbf{B}_{0} \mathbf{C}_{0}: \text { Testigo } \\
\mathbf{B}_{1} \mathbf{C}_{0}: \text { Fertilizante } \\
\mathbf{B}_{0} \mathbf{C}_{1} \text { Herbicida } \\
\mathbf{B}_{1} \mathbf{C}_{1}: \text { Fert }- \text { Herb }\end{array}$ & $\begin{array}{l}\text { 5) } \mathrm{Su} \\
\text { 6) } \mathrm{Su} \mathrm{Fe} \\
\text { 7) } \mathrm{Su} \mathrm{He} \\
\text { 8) } \mathrm{Su} \mathrm{Fe} \mathrm{He}\end{array}$ & $\begin{array}{l}45 \\
55 \\
60 \\
81\end{array}$ & $\begin{array}{l}63 \\
63 \\
93 \\
95\end{array}$ & $\begin{array}{l}0.29 \\
0.32 \\
0.40 \\
0.40\end{array}$ & $\begin{array}{l}0.5 \\
0.6 \\
0.7 \\
0.6\end{array}$ & $\begin{array}{r}9.73 \\
12.50 \\
25.63 \\
19.44\end{array}$ \\
\hline TOTAL $A_{1}$ & & & 60 & 78 & 0,35 & 0,6 & 16.95 \\
\hline$A_{2}$ Subsolado & $\begin{array}{l}\mathrm{B}_{0} \mathrm{C}_{0}: \text { Testigo } \\
\mathrm{B}_{1} \mathrm{C}_{0} \text { Fertilizante } \\
\mathrm{B}_{0} \mathrm{C}_{1} \text { Herbicida } \\
\mathrm{B}_{1} \mathrm{C}_{1}: \text { Fert }- \text { Herb }\end{array}$ & $\begin{array}{l}\text { 9) } \mathrm{Sb} \\
\text { 10) } \mathrm{Sb} \mathrm{Fe} \\
\text { I1) } \mathrm{Sb} \mathrm{He} \\
\text { 12) } \mathrm{Sb} \mathrm{Fe} \mathrm{He}\end{array}$ & $\begin{array}{l}52 \\
36 \\
\times 5 \\
81\end{array}$ & $\begin{array}{l}80 \\
37 \\
99 \\
95\end{array}$ & $\begin{array}{l}0.27 \\
0.28 \\
0.40 \\
0.56\end{array}$ & $\begin{array}{l}0.5 \\
0.6 \\
0.8 \\
0.9\end{array}$ & $\begin{array}{r}6.92 \\
9.40 \\
30.16 \\
64.13\end{array}$ \\
\hline TOTAL $A_{2}$ & & & 63 & 78 & 0.38 & 0,7 & 27,63 \\
\hline $\begin{array}{l}\text { TOTALES } \\
\text { FACTORES } \\
\text { B y C } \\
\text { (Todos los mé- } \\
\text { de prep. de suelo) }\end{array}$ & $\begin{array}{l}\mathrm{B}_{0} \mathrm{C}_{0} \text {. Testigo } \\
\mathrm{B}_{1} \mathrm{C}_{0} \text {. Fertilizante } \\
\mathrm{B}_{0} \mathrm{C}_{1} \text { : Herbicida } \\
\mathrm{B}_{1} \mathrm{C}_{1} \text {. Fert - Herb }\end{array}$ & $\begin{array}{rrr}1 & -5 & -9 \\
2 & -6 & -10 \\
3 & -7 & -11 \\
4 & 8 & -12\end{array}$ & $\begin{array}{l}49 \\
52 \\
81 \\
85\end{array}$ & $\begin{array}{l}67 \\
55 \\
97 \\
97\end{array}$ & $\begin{array}{l}0.28 \\
0.31 \\
0.43 \\
0.52\end{array}$ & $\begin{array}{l}0.5 \\
0.6 \\
0.8 \\
0.8\end{array}$ & $\begin{array}{l}7.77 \\
10.42 \\
30.43 \\
46.31\end{array}$ \\
\hline
\end{tabular}

NOTA (1): Se incluye replante al primer año./ Ho: Hoyo: Su: Surco: Sb: Subsolado: Fe: Fertilizante; He: Herbicida. 


\section{CUADRO 3}

NIVELES DE SIGNIFICANCIA DE LOS DISTINTOS FACTORES Y SU INTERACCION PARA LA PLANTACION EXPERIMENTAL DE Eucalyptus camaldulensis (EDAD 2 ANOS)

\begin{tabular}{|c|c|c|c|c|c|}
\hline \multirow[t]{2}{*}{ Factor } & \multicolumn{2}{|c|}{ Supervivencia } & \multirow[t]{2}{*}{ Altura (1) } & \multirow{2}{*}{$\begin{array}{l}\text { Diámetro } \\
\text { Basal (1) }\end{array}$} & \multirow{2}{*}{$\begin{array}{c}\text { Indice de } \\
\text { Crecimiento } \\
\text { (1) }\end{array}$} \\
\hline & Sin replante & Con replante & & & \\
\hline Bloques . & $* *$ & - & * & $* *$ & * \\
\hline$A=$ Preparación del suelo & . & * & . & . & - \\
\hline $\mathrm{B}=$ Fertilizante & - & . & * & * & * \\
\hline $\mathrm{C}=$ Control de competencia & ** & ** & ** & $* *$ & $* *$ \\
\hline A.B & * & - & - & - & - \\
\hline A.C & - & - & - & - & - \\
\hline B.C & - & - & * & * & * \\
\hline A.B.C & - & . & - & - & - \\
\hline
\end{tabular}

NOTA (1): Con replante: $\left({ }^{*}\right)$ Significativo al $95 \%$ prob.: $\left(^{*}\right.$ ) Significativo al $95 \%$ y $99 \%$ prob.

\section{CUADRO 4}

\section{NIVELES DE SIGNIFICANCIA DE LOS DISTINTOS FACTORES Y SU INTERACCION PARA LA PLANTACION EXPERIMENTAL DE Quillaja saponaria (EDAD 2 AÑOS).}

\begin{tabular}{|c|c|c|c|c|c|}
\hline \multirow[t]{2}{*}{ Factor } & \multicolumn{2}{|c|}{ Supervivencia } & \multirow[t]{2}{*}{ Altura (1) } & \multirow{2}{*}{$\begin{array}{l}\text { Diámetro } \\
\text { Basal (1) }\end{array}$} & \multirow{2}{*}{$\begin{array}{l}\text { Indice de } \\
\text { Crecimiento } \\
\text { (1) }\end{array}$} \\
\hline & Sin replante & Con replante & & & \\
\hline Bloques & $* *$ & $* *$ & $* *$ & $* *$ & $* *$ \\
\hline $\mathrm{A}=$ Preparación del suclo & $* *$ & - & - & - & - \\
\hline $\mathrm{B}=$ Fertilizante & - & - & * & - & - \\
\hline $\mathrm{C}=$ Control de competencia & ** & $* *$ & ** & ** & $* *$ \\
\hline A.B & - & - & - & - & . \\
\hline A.C & $*$ & - & - & - & - \\
\hline B.C & $* *$ & $* *$ & - & - & . \\
\hline A.B.C & - & - & - & - & - \\
\hline
\end{tabular}

NOTA (1): Con replante; $\left({ }^{*}\right)$ Significativo al $95 \%$ prob.: $\left({ }^{*}{ }^{*}\right)$ Significativo al $95 \%$ y $99 \%$ prob. 
FIGURA 1

SUPERVIVENCIA

E. camaldulensis (2 años)
FIGURA 2

CRECIMIENTO

E. camaldulensis (2 años)

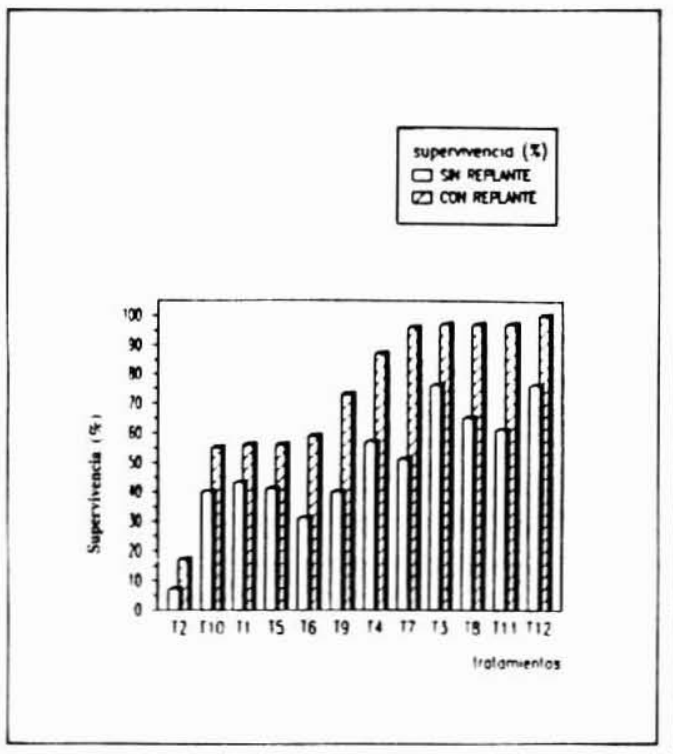

FIGURA 3

SUPERVIVENCIA

Q. saponaria (2 años)

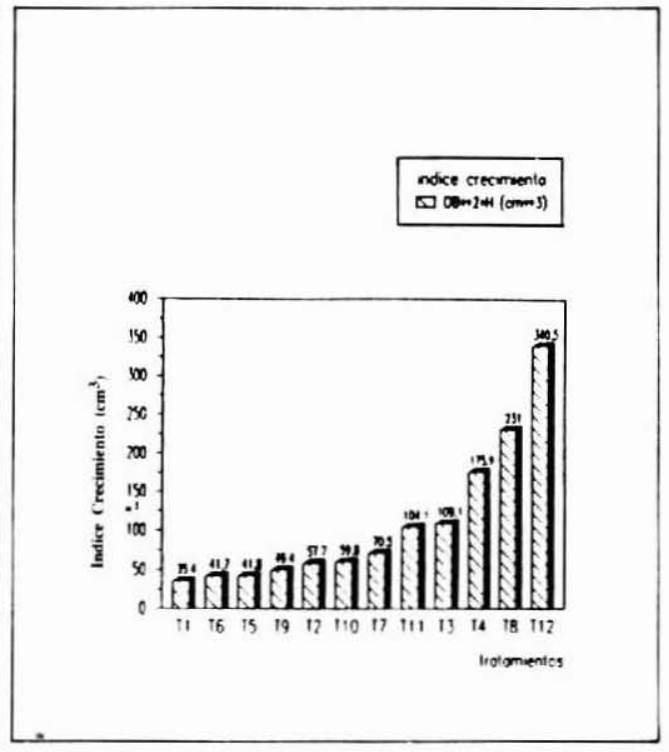

FIGURA 4

CRECIMIENTO

Q. saponaria (2 años)
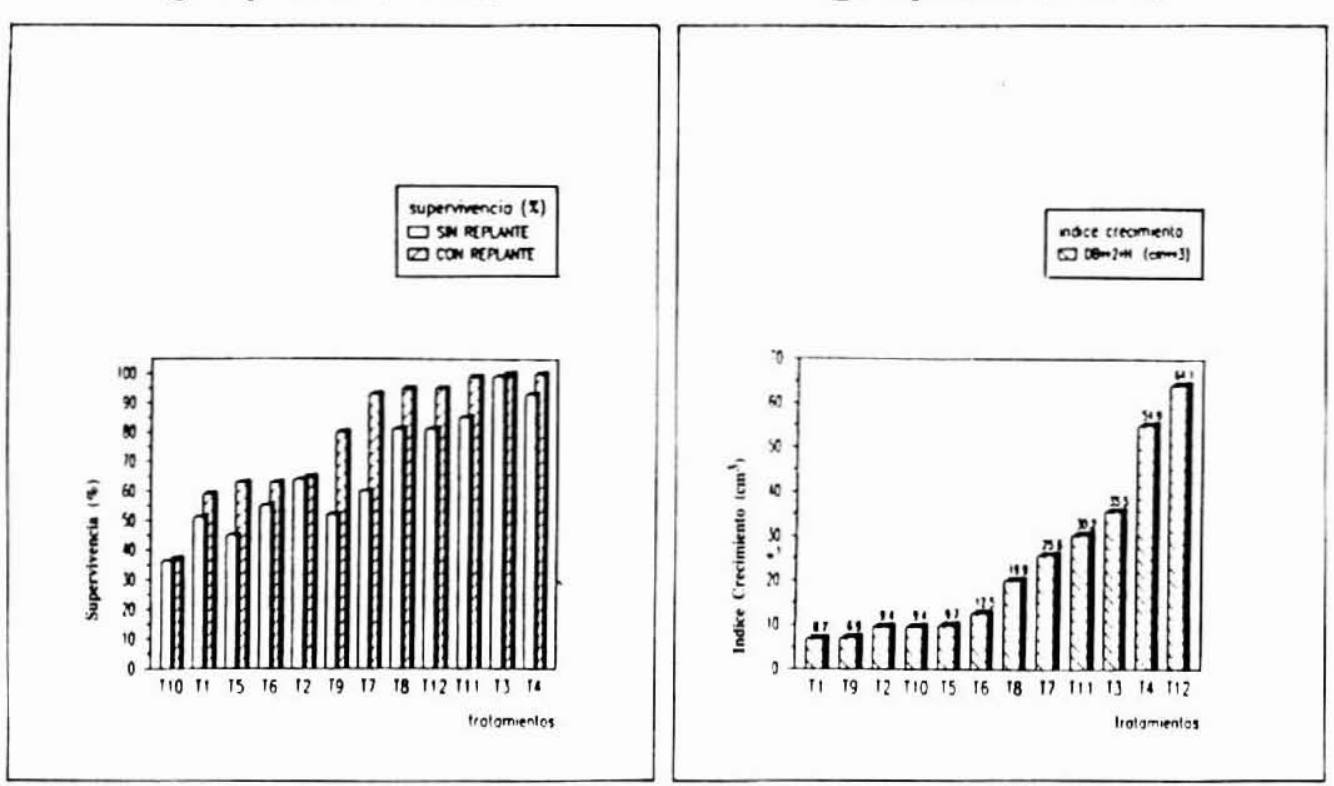
El factor aislado más importante en la respuesta de la supervivencia y el crecimiento es el control de competencia (Cuadros 3 y 4) o control de malezas. El otro factor de mayor importancia es el sitio (bloques). La diferencia de supervivencia y crecimiento entre los bloques se debe al crecimiento menor de las plantas en el bloque expuesto al viento S.W.

Es interesante destacar que gracias al replante del primer año con Eucalyptus camaldulensis, este último factor pudo neutralizarse. En menor grado influyeron la fertilización y la interacción fertilizante - control de competencia.

Es importante destacar que la fertilización por sí sola presenta un efecto negativo en el crecimiento y la supervivencia, puesto que los nutrientes son aprovechados por la vegetación competidora. Es así como la interacción de los tratamientos fertilizante - control de competencia, presentaron bondades significativas en el crecimiento de Eucalyptus camaldulensis y en supervivencia de Quillaja saponaria.

La preparación de suelo presenta una baja significación. Sin embargo, al combinar el tratamiento subsolado con aplicación de fertilizante y control de competencia se obtuvo el mejor resultado en crecimiento. (Figuras 5 y 6 ).

\section{DISCUSION}

Dos años después del establecimiento de estas plantaciones se pueden observar resultados interesantes para ambas especies, a pesar del replante que fue necesario efectuar al cumplirse el primer año. Como se anotó anteriormente, los resultados en general se vieron afectados por la sequía del año de plantación (1985) y el viento que afectó a algunas parcelas. Se analizan a continuación los efectos de cada uno de los factores en la supervivencia y el crecimiento inicial de las plantas.

\section{Preparación del Suelo}

En ambas especies ensayadas, la supervivencia mejoró con la preparación del suelo con subsolado (Cuadros 1, 2). Este hecho es de importancia, en consideración a las dificultades de la plantación en las zonas áridas.

El crecimiento inicial no mejoró con las diferentes intensidades de preparación del suelo sin considerar los otros factores. De acuerdo a experiencias con especies de rápido crecimiento, una preparación intensiva del suelo representa una ganancia significativa en crecimiento y por lo tanto en ingresos (SCHONAU, 1984 a; BODEN, 1984). Se espera que la respuesta se manifieste dentro de los próximos años. (BODEN, D.I. 1984) en una experiencia con Acacia mearnsii y Eucalyptus grandis determinó que las plantas presentan una respuesta extraordinariamente favorable al subsolado, especialmente si además se fertiliza. Esto también se observó en el presente estudio para Quillaja saponaria y Eucalyptus camaldulensis, donde la aplicación de herbicidas y fertilizantes tiene el mejor resultado en las parcelas preparadas son subsolado. 
FIGURA 5

CRECIMIENTO E. camaldulensis SEGUN TRATAMIENTO SUELO (2 años)

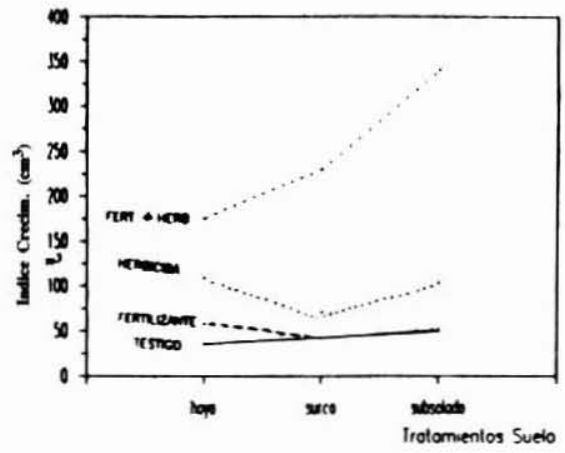

FIGURA 7

CRECIMIENTO $E$. camaldulensis PARA TODOS LOS TRATAMIENTOS (2 años)

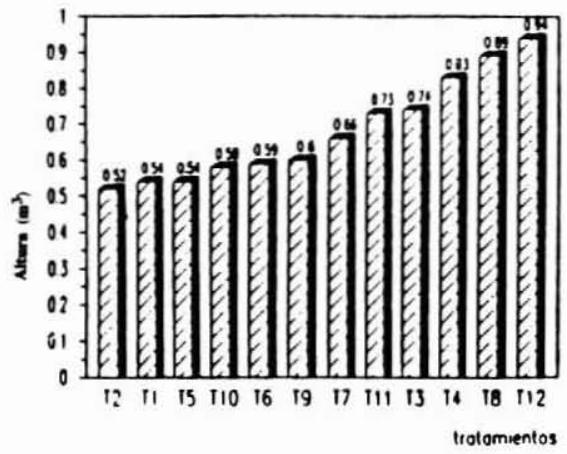

FIGURA 6 CRECIMIENTO Q. saponaria SEGUN TRATAMIENTO SUELO (2 años)

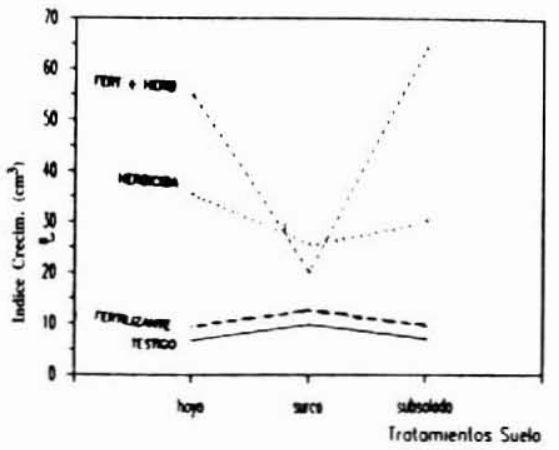

FIGURA 8

CRECIMIENTO $Q$. saponaria PARA TODOS LOS TRATAMIENTOS (2 años)

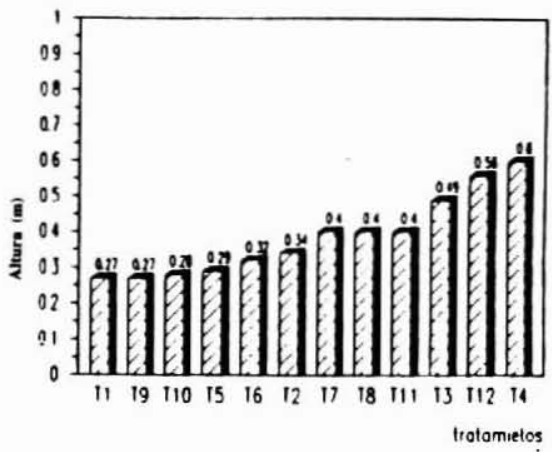




\section{Control de Competencia}

El control de competencia resultó ser el factor de mayor importancia en el establecimiento de ambas especies, permitiendo una supervivencia significativamente mayor, incluyendo o no un replante. También en el crecimiento inicial, el factor que más gravita en el éxito del establecimiento es el control de competencia.

El control de competencia ha sido una práctica poco usual en el establecimiento de plantaciones forestales en la zona árida o semiárida.

Los resultados de este ensayo y de otro en la zona central (PRADO y ROJAS, 1987) están demostrando que es importante su incorporación. SCHONAU (1984 a) destaca la importanciá de usar herbicidas en el establecimiento de Eucalyptus grandis, como una alternativa en suelos cuya pendiente no permite una preparación intensiva de suelo. En experiencias en la zona semiárida de Chile, se determinó que una buena práctica es el control manual (raspado del suelo en círculos de $1 \mathrm{~m}$ alrededor de la planta). Este resultó comparable con la aplicación de herbicidas, tomando como base un ensayo con 16 productos y/o dosis diferentes (AGUIRRE, S., 1987).

\section{Fertilización}

La fertilización por sí sola no tiene un efecto beneficioso en el establecimiento de la plantación. Se puede observar que, independientemente de la preparación del suelo, las parcelas fertilizadas presentan la menor sobrevivencia. Esto se debe al aprovechamiento que hace la vegetación competidora del fertilizànte y del agua del suelo circundante. El crecimiento inicial tampoco se ve favorecido aplicando el fertilizante; en cambio, se produce una respuesta muy favorable cuando se hace además un control de la maleza y una buena preparación de suelo,

Es interesante hacer resaltar la importancia de la fertilización adecuada. Trabajos en el extranjero con Eucalyptus globulus mostraron aumentos de producción de $80 \mathrm{~m} 3 /$ ha al término de la rotación, agregando NPK (VIEIRA DE BRITO, 1979, y CROMER and WILLIAMS, 1982, citado en SCHONAU 1984 b). En dichas experiencias la cantidad de nitrógeno, fósforo y potasio (NPK) aplicado por hectárea fue de 200 - 300; 52 - 90; cero a $149 \mathrm{Kg}$, respectivamente. En esta experiencia de Illapel, considerando un espaciamiento de la plantación de $3 \mathrm{~m} \times 3 \mathrm{~m}$, la cantidad de NPK aplicado por ha es de $112 \mathrm{Kg}, 22 \mathrm{Kg}$ y $55 \mathrm{Kg}$, respectivamente. Debido a que en el lugar de este ensayo no existen deficiencias de estos nutrientes (TORO, J., 1986), se puede esperar que la respuesta en términos absolutos sea significativa (SCHONAU, 1984 b). Por otra parte, la fertilización abre posibilidades de incorporar terrenos marginales con deficiencias de nutrientes, al uso forestal.

\section{Otros Factores}

Este ensayo demostró la importancia que tiene la elección del lugar a plantar. Como se mencionó anteriormente, un bloque del ensayo de $\boldsymbol{E}$. camaldulensis y de $\boldsymbol{Q}$. saponaria quedaron expuestos al fuerte viento S.W., afectando notoriamente la supervivencia y el desarrollo inicial de esas plantas. Es por lo tanto del mayor interés elegir cuidadosamente los lugares a forestar en las zonas áridas, lo que significa en la práctica la imposibilidad de forestar paños completos, debiendo restringirse a lugares más protegidos que puedan conservar la escasa humedad de aquellas áreas. 
Indudablemente el riego inicial y la cantidad de lluvias caídas por año tienen una enorme importancia en los resultados. El año seco (1985) significó pérdida en el primer año, así como el replante en invierno 1986 se benefició con la mayor precipitación caída en ese año.

\section{CONCLUSIONES}

Los resultados obtenidos con este ensayo permiten concluir lo siguiente:

- El control de la competencia es el factor más importante para asegurar la supervivencia y permitir un buen desarrollo inicial en el establecimiento de ambas especies estudiadas.

- La fertilización es recomendable, solamente si se acompaña con un control de malezas. De otra manera tiene un efecto negativo en la supervivencia, al favorecer la vegetación competidora.

- El mejor tratamiento es la combinación de preparación de suelo con subsolado, control de la maleza y aplicación de fertilizante.

- Los sectores muy expuestos a vientos fuertes, no son recomendables para la plantación con las especies ensayadas.

\section{AGRADECIMIENTOS}

Esta experiencia forma parte de las actividades desarrolladas en el proyecto "Regeneración Forestal", financiado por el Centro Internacional de Investigaciones para el Desarrollo de Canadá (CIID).

Este ensayo se basa en un diseño elaborado por el Sr. José A. Prado, Jefe de la División Silvicultura de INFOR.

Los autores agradecen la revisión crítica y recomendaciones realizadas por el Ing. Forestal de INFOR, Sr. Hans Grosse. 


\section{REFERENCIAS BIBLIOGRAFICAS}

1. BACKSTROM. 1981. Site preparation and direct seeding in Swedish Forestry. In: Forest Regeneration. Proc. Symposium on Engineering Systems for Forest Regeneration. American Society of Agricultural Eng.. Michigan. U.S.A. pp. $208-216$

2. BODEN. 1). I. 1984 Early response to different methods of site preparation for three commercial species. In: Proc: IUFRO Symposium on site and productivity of fast growing plantations. Pretoria and Pietermaritzburg. South Alrica. pp $565-578$.

3. (ORFO - INFOR (Corporación de Fomento de la Producción - Instituto Forestal). 1986: Especies Forestales Exóticas de Interés económico para Chile. CORFO. Gerencia de Desarrollo. AF 86/32, Santiago. 168 p.

4. CROMER. R.N. 1984. Site ameloration for fast growing plantations. CSIRO. Australia. In: Proc. IUFRO Symposium on Site and Productivity of fast growing plantations, May 1984, Pretoria and Pietermaritzburg. South, Africa. Vol. I. pp 181 - 196.

5. DI CASTRI, F. y HAJEK. E.R. 1976. Bioclimatologia de Chile. Santiago. Chile. Universidad Católica de Chile. $128 \mathrm{p}$.

6. FlenZALIDA. H. 1967. Climatología de Chile. En: Corporación de Fomento de la Producción: Geografía Económica de Chile. pp. 99- 152.

7. PRADO. J.A. y ROJAS, P. 1987. Preparación del sitio y fertilización en el establecimiento de plantaciones de Eucalyptus globulus en la zona semiárida de Chile. Ciencia e Investigación Forestal (1) 17 -27. INFOR. Santiago.

8. SCHONAC, A.P.G. y VERLOREN VAN THEMAAT, R. and BOIDN. D. I. 1981. The importance of complete site preparation and fertilizing in the establishment of Eucalyptus grandis. S. Afr. For. J. 116: 1 - 10.

9. SCHONAU. A. P. G. 1984 a. Silvicultural considerations for high productivity of Eucalyptus grandis. Forest Ecology and Management. $9(1984) 295$ - 314.

10. SCHONAL. A. P. G. 1984 b. Fertilization of fast growing broadleaved species. In: Proc. IUFRO Symposium on site and productivity of fast growing plantations. Pretoria and Pietermaritzburg. South Africa. pp. 253 -268.

11. TORO. J. 1986. Descripción de suelos e instalación de ensayos de fertilización en plantaciones de eucalyptus. Informe Final Instituto Forestal. Santiago, I8 p. 